Article

\title{
Detection of a Conspecific Mycovirus in Two Closely Related Native and Introduced Fungal Hosts and Evidence for Interspecific Virus Transmission
}

\author{
Corine N. Schoebel *, Simone Prospero ${ }^{D}$, Andrin Gross and Daniel Rigling \\ Swiss Federal Institute for Forest, Snow and Landscape Research, WSL, Zuercherstrasse 111, \\ 8903 Birmensdorf, Switzerland; Simone.Prospero@wsl.ch (S.P.); andrin.gross@wsl.ch (A.G.); \\ Daniel.rigling@wsl.ch (D.R.) \\ * Correspondence: corine.schoebel@wsl.ch; Tel.: +41-44-739-25-28
}

Received: 24 October 2018; Accepted: 10 November 2018; Published: 13 November 2018

check for updates

\begin{abstract}
Hymenoscyphus albidus is a native fungus in Europe where it behaves as a harmless decomposer of leaves of common ash. Its close relative Hymenoscyphus fraxineus was introduced into Europe from Asia and currently threatens ash (Fraxinus sp.) stands all across the continent causing ash dieback. H. fraxineus isolates from Europe were previously shown to harbor a mycovirus named Hymenoscyphus fraxineus Mitovirus 1 (HfMV1). In the present study, we describe a conspecific mycovirus that we detected in H. albidus. HfMV1 was consistently identified in H. albidus isolates (mean prevalence: $49.3 \%$ ) which were collected in the sampling areas before the arrival of ash dieback. HfMV1 strains in both fungal hosts contain a single ORF of identical length (717 AA) for which a mean pairwise identity of $94.5 \%$ was revealed. The occurrence of a conspecific mitovirus in H. albidus and $H$. fraxineus is most likely the result of parallel virus evolution in the two fungal hosts. HfMV1 sequences from $H$. albidus showed a higher nucleotide diversity and a higher number of mutations compared to those from $H$. fraxineus, probably due to a bottleneck caused by the introduction of H. fraxineus in Europe. Our data also points to multiple interspecific virus transfers from H. albidus to $H$. fraxineus, which could have contributed to the intraspecific virus diversity found in $H$. fraxineus.
\end{abstract}

Keywords: Chalara fraxinea; Hymenoscyphus pseudoalbidus; ash dieback; Narnaviridae; evolution; invasive species; horizontal virus transmission

\section{Introduction}

In the past years, species invasions have become an important topic in the scientific literature due to increasing awareness of the consequences of intensified international trade of plant material [1-3]. In the case of introduced (exotic) plant pathogens, we frequently observe invasions by species that have a harmless close relative in the invaded area and which are often more virulent due to the lack of co-evolution with the novel host. This can have catastrophic impacts on forest ecosystems (e.g., $[4,5])$. So far, most studies have focused on the invasive species itself, while little attention has been paid to possible interactions with closely related native species that may occupy similar ecological niches and share evolutionary histories. The duration of this interaction is often limited because the invasive species outcompetes its native sister species (e.g., Cryphonectria parasitica vs. C. radicalis in Europe; [6]). Nevertheless, during this short timeframe, gene flow between native and introduced species may occur, resulting in varying levels of gene introgression between the two interacting taxa. The occurrence of hybridization between allopatric species following attained sympatry because of anthropogenic introduction has been reported for example in the basidiomycete genus Heterobasidion [7] and in the oomycete genus Phytophthora [8]. 
In the present study we focus on the interaction of Hymenoscyphus fraxineus and $H$. albidus, which belong to the family Helotiaceae and are closely related [9]. The first species is native to Asia and in Europe is an invasive pathogen causing ash dieback (ADB), mainly on common (Fraxinus excelsior) and narrow-leaved ash (Fraxinus angustifolia) [10]. The latter species is a native endophyte of common ash in Europe (e.g., [11]). Due to its severe ecological consequences, there has lately been a great interest in the biology, ecology, and population genetics of $H$. fraxineus (e.g., [12-16]). However, little is known about $H$. albidus $[17,18]$, as well as its interaction with $H$. fraxineus. Field reports indicate that since the onset of the ADB epidemic in Europe, the frequency of detection of $H$. albidus has decreased strongly, with the species being at risk of local extinction $[19,20]$. Both species show a similar life cycle and, more importantly, occupy the same sporulation niche, i.e., rachis and petioles of last year's ash leaves in the litter $[17,18,20]$. The introduced $H$. fraxineus is most likely outcompeting its native relative because of its superior ability to cause leaf infections and its massive production of airborne ascospores [21]. After the first detection of the disease in the 1990s in Eastern Europe [22], H. fraxineus is now abundant in most ash stands all across mainland Europe and the UK $[15,23,24]$.

Fungal viruses (mycoviruses) are widespread in all major groups of fungi [25] and a mycovirus, Hymenoscyphus fraxineus Mitovirus 1 (HfMV1) was recently also discovered in $H$. fraxineus [26]. HfMV1 is a putative member of the genus Mitovirus (family Narnaviridae) that are only present in fungi. Mitoviruses are commonly found in fungi and represent the simplest mycoviruses with an unencapsidated single strand RNA genome of approximately $2.5 \mathrm{~kb}$. The genome contains one single open reading frame (ORF), encoding the RNA-dependent RNA polymerase (RdRp). As many other RNA viruses, mitoviruses are mutating at high rates [27]. Mitoviruses are located and translated in the mitochondria $[28,29]$. It has previously been shown that some mycoviruses may be transferred between related fungal species e.g., in the genera Cryphonectria, Heterobasidion, Sclerotinia, as well as between fungal taxa e.g., Sclerotinia homoeocarpa and Ophiostoma novo-ulmi [30-33]. In many of these studies, evidence for interspecific virus transmission was based on the occurrence of conspecific viruses in different fungal host species.

In the present study, we tested the occurrence of a conspecific mitovirus in $H$. albidus and $H$. fraxineus using the RdRP gene as a marker for population genetic analyses. Specifically, we (i) determined the presence and prevalence of HfMV1 sequences in H. albidus isolates, which were collected in Switzerland and France before the arrival and spread of the invasive H. fraxineus; (ii) determined the phylogenetic relationship of the viral sequences detected in $H$. albidus and $H$. fraxineus; and (iii) compared the genetic diversity of the viruses in $H$. albidus and $H$. fraxineus populations from the same geographic regions, to determine the possible origin of the conspecific virus in the two closely related host species.

\section{Materials and Methods}

\subsection{Isolates Used in the Study}

Overall, 67 isolates of $H$. albidus from France and Switzerland and 221 HfMV1-positive isolates of H. fraxineus from Europe (mainly Switzerland) were analyzed (Table 1). All H. albidus isolates were recovered from colonized petioles of common ash. The $H$. fraxineus isolates were also obtained from common ash, either from petioles or bark lesions (for details see [14,26]). All H. albidus isolates were identified by ITS sequencing using ITS1 and ITS4 primers [34]. The H. fraxineus isolates were identified either by ITS sequencing or microsatellite genotyping [12] 
Table 1. Overview of the Hymenoscyphus albidus and H. fraxineus isolates included in the present study.

\begin{tabular}{|c|c|c|c|c|c|c|}
\hline Host & Region (Abbreviation) & $\begin{array}{l}\text { Canton } \\
\text { (s)/Region }\end{array}$ & $\begin{array}{c}\text { Number of } \\
\text { Samples Screened }\end{array}$ & $\begin{array}{c}\text { Number of Samples } \\
\text { with HfMV1 }\end{array}$ & Sampling Year (s) & $\begin{array}{l}\text { Reference for Fungal } \\
\text { Isolates }\end{array}$ \\
\hline H. albidus & & & 67 & 34 & & \\
\hline \multirow[t]{3}{*}{ Switzerland } & South-Western Switzerland (SW-CH) & VS, VD & 13 & 13 & $2008,2009,2012$ & This study \\
\hline & Southern Switzerland (S-CH) & TI & 33 & 12 & $2009,2010,2012$ & [35]; this study \\
\hline & Northern Switzerland (N-CH) & GL, ZH & 7 & 4 & 2009 & [35] \\
\hline France & North-Western France (NW-F) & Bretagne & 14 & 5 & 2012 & [17]; this study \\
\hline H. fraxineus & & & ** & 221 & & \\
\hline \multirow[t]{3}{*}{ Switzerland } & South-Western Switzerland (SW-CH) & VS, VD & $* *$ & 41 & 2013, 2016 & [13]; this study \\
\hline & Southern-Switzerland * $(\mathrm{S}-\mathrm{CH})$ & TI, GR & $* *$ & 90 & $2014,2013,2016$ & [15]; this study \\
\hline & Northern-Switzerland (N-CH) & SG, SZ, UR & ** & 85 & 2013,2014 & [13]; this study \\
\hline
\end{tabular}

* Including two HfMV1 positive isolates from Northern Italy; ** HfMV1 positive isolates were selected, no data on number initially screened. 


\subsection{Fungal Cultivation and RNA Extraction}

For each sample, a small piece of growing pure culture was transferred to a new cellophane covered malt agar plate [13]. These were incubated for 5 weeks in the dark at room temperature. Thereafter, the mycelia were harvested, lyophilized and then milled in a MixerMill (MM33, Retsch $\mathrm{GmbH}$, Haan, NRW, Germany). For each sample, approximately $20 \mathrm{mg}$ of the ground mycelium were used for RNA extraction, using the PureLink Pro 96 total RNA Purification Kit (Invitrogen, Carlsbad, CA, USA), following the manufacturer's instructions.

\subsection{Screening for HfMV1 and Viral Sequencing}

All $H$. albidus and $H$. fraxineus isolates were screened for the presence of HfMV1 using a specific PCR assay [26]. Complementary DNA (cDNA) was produced with the Maxima First Strand cDNA Synthesis Kit (Life Technologies, Carlsbad, CA, USA) according to the manufacturer's protocol. For sequencing, PCR products were purified using Illustra ExoProStar (GE Healthcare Life Sciences, Pittsburgh, PA, USA) according to the instructions. Sanger sequencing of the PCR products was done on both strands using the same primers as for PCR. In addition, for 40 isolates (8 for H. albidus, 32 for $H$. fraxineus) the full length viral RdRP gene (2151 bp) was sequenced in both directions as described in [26]. To account for mutations at primers sites in some of the isolates, additional primers had to be designed to obtain the complete ORF (see Table S2).

\subsection{Phylogenetic Analyses}

The maximum likelihood (ML) phylogenetic analysis was conducted for the nucleotide alignment. The alignment quality was first double-checked using the MACSE aligner [36]. A total of 40 separate runs each with 10,000 replicates were conducted using the 'rapid bootstrap analysis and tree search' algorithm, resulting in a best ML tree with branch lengths and bootstrap support values. Two separate analyses were performed, (i) for the full length RdRP gene (2151 bp, N = 40) and (ii) for the partial RdRP gene (495 bp, $N=255$ ). For the partial RdRP gene, three distinct data partitions with joint branch length optimization in RAxML v8.2.4 [37] implementing the GTRGAMMA model of nucleotide substitution were used.

The Bayesian analysis was conducted for each of the two datasets separately, using MRBAYES v3.2.6 [38]. Three independent runs, each consisting of 100 million generations with a burn-in of 80 million and a sampling frequency of 1000 were performed.

The resulting data was visualized using the software TREEGRAPH v2 [39] and FIGTREE v1.4.2 (http://tree.bio.edac.uk/software/figtree/).

\subsection{Genetic Diversity, Differentiation and Evolution}

DNASP v5 [40] was used to determine the nucleotide diversity estimated by the average number of differences per site between two sequences $(\pi)$, the number of haplotypes observed (h), the haplotype diversity (Hd), the number of segregating sites $(S)$, the number of total mutations $(\eta)$, and the average number of differences $(\mathrm{K})$ per country/population were calculated for the two datasets 'partial ORF' and 'full ORF'. Pairwise identity percentages for the nucleotide and amino acid sequences were calculated using Multiple Sequence Comparison by Log- Expectation (MUSCLE; http:/ /www.ebi.ac. uk/Tools/msa/muscle).

\section{Results}

In the present study, we describe the first fungal virus detected in H. albidus. HfMV1, which was previously described for $H$. fraxineus, could now also be detected in its close relative, $H$. albidus. Although vast areas of Switzerland were sampled, from 2013 onward H. albidus could not be isolated any longer. 


\subsection{Prevalence of HfMV1 in H. albidus}

All H. albidus isolates analysed originated from Swiss and French regions where H. fraxineus had not been officially reported at the time of sampling (Figure 1). By using a HfMV1-specific PCR assay, we detected HfMV1-sequences in 33 out of $67 \mathrm{H}$. albidus isolates (49.3\%). More precisely, 28 out of 53 Swiss isolates (52.8\%) and 5 out of 14 French isolates $(35.7 \%)$ harbored HfMV1.

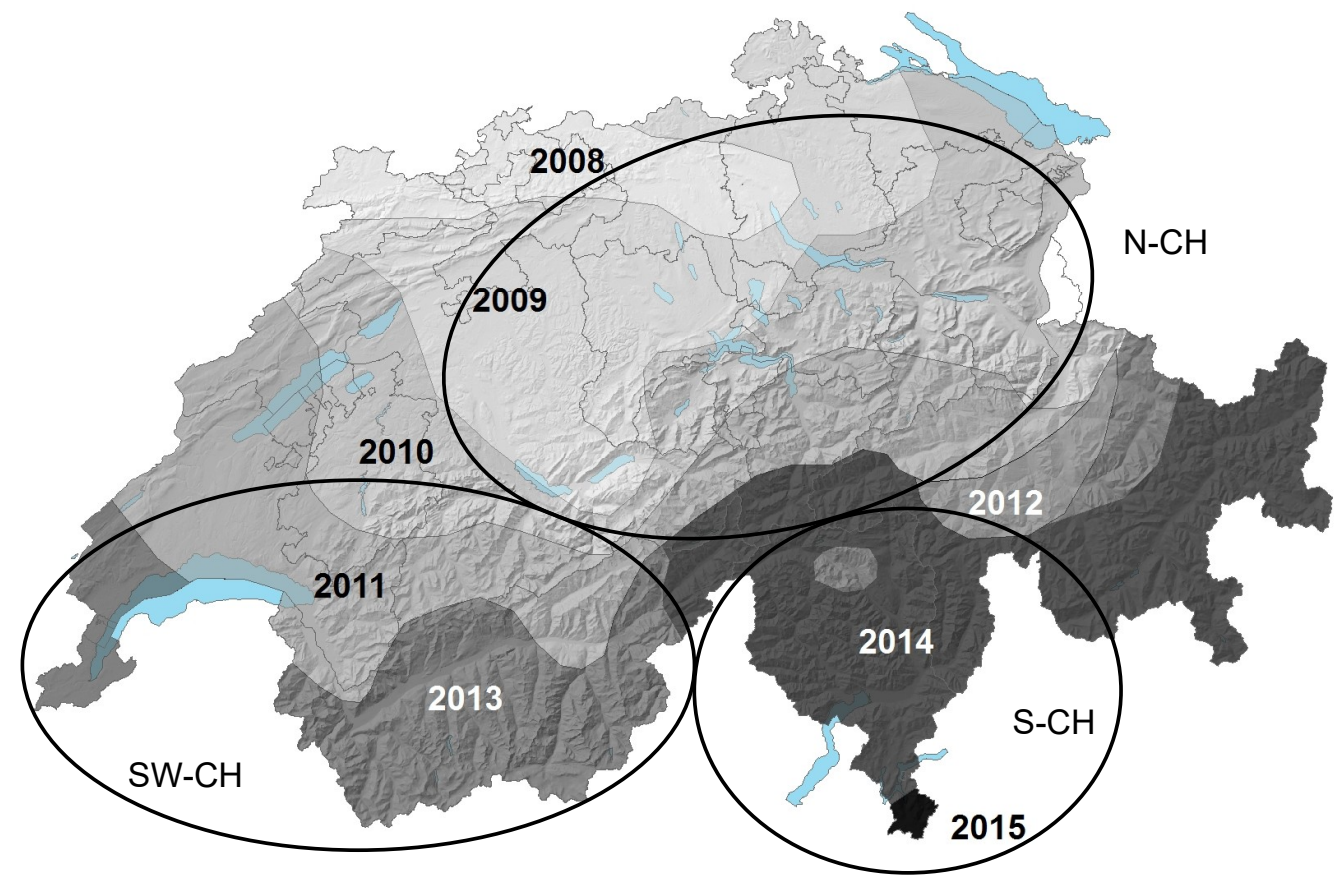

Figure 1. Geographic origin of the Swiss Hymenoscyphus albidus and H. fraxineus samples analyzed in this study. Grey tones display the year of first report of ash dieback. Light grey lines display Swiss canton borders. Abbreviations: S-CH: Southern Switzerland, SW-CH: South-Western Switzerland, $\mathrm{N}-\mathrm{CH}$ : Northern Switzerland. Modified after [41].

\subsection{Pairwise Sequence Identity}

All virus sequences obtained from $H$. albidus and $H$. fraxineus showed high similarities and were of identical length for both the full and partially sequenced RdRP gene. Average pairwise sequence identity (PWI) was 95\% (range between 93\% to 97\%), when comparing the full ORF amino acid sequences (717 AA) of HfMV1 originating from the two fungal host species. For the nucleotide sequences (NT) the average PWI was 91\% (90\% to 93\%). When considering only HfMV1 sequences from $H$. albidus, the PWI value was on average 97\% (92\% for NT), whereas for HfMV1 sequences from $H$. fraxineus, the PWI value was $98 \%$ (96\% for NT). According to the International Committee on Taxonomy of Viruses, a PWI $<40$ indicates different species, whereas PWI $>90$ is indicative that the compared sequences belong to the same virus species [42]. Therefore, we can conclude that all viral sequences analyzed in this study belong to the same species, which was previously described as HfMV1 [26].

\subsection{Phylogenetic Relationships of HfMV1 across Two Different Host Species}

For the full RdRP gene of HfMV1 (2151 bp), three well supported groups of sequences were detected (posterior probability, PP = 1; Figure 2). RAxML analysis evidenced very similar three groups, with $>90 \%$ bootstrap support (Figure S1). In accordance with [15], these groups were called HfMV1 group 1, HfMV1 group 2 and HfMV1 H. albidus group. All viral sequences obtained from $H$. albidus belonged to a separate group, which consisted of long-branched sequences. HfMV1 group 2 also included long-branched sequences, all from Switzerland. HfMV1 group 1, on the other hand, was 
characterized by short branches with little differentiation and low support values. The sequences of the German and Polish isolates, which were initially used for HfMV1 primer design [26], came to reside in this group (Hf_DE_C436 and Hf_PL_C428 in Figure 2). The full RdRP sequences from H. fraxineus were allocated both to HfMV1 group 1 (11 isolates, light grey in Figure 2) and HfMV1 group 2 (19 isolates, black in Figure 2). All 8 full RdRP sequences obtained from H. albidus formed the H. albidus group. In addition, also two full RdRP sequences of $H$. fraxineus isolates obtained from necrotic lesions cluster here (arrows and blue font in Figure 2).

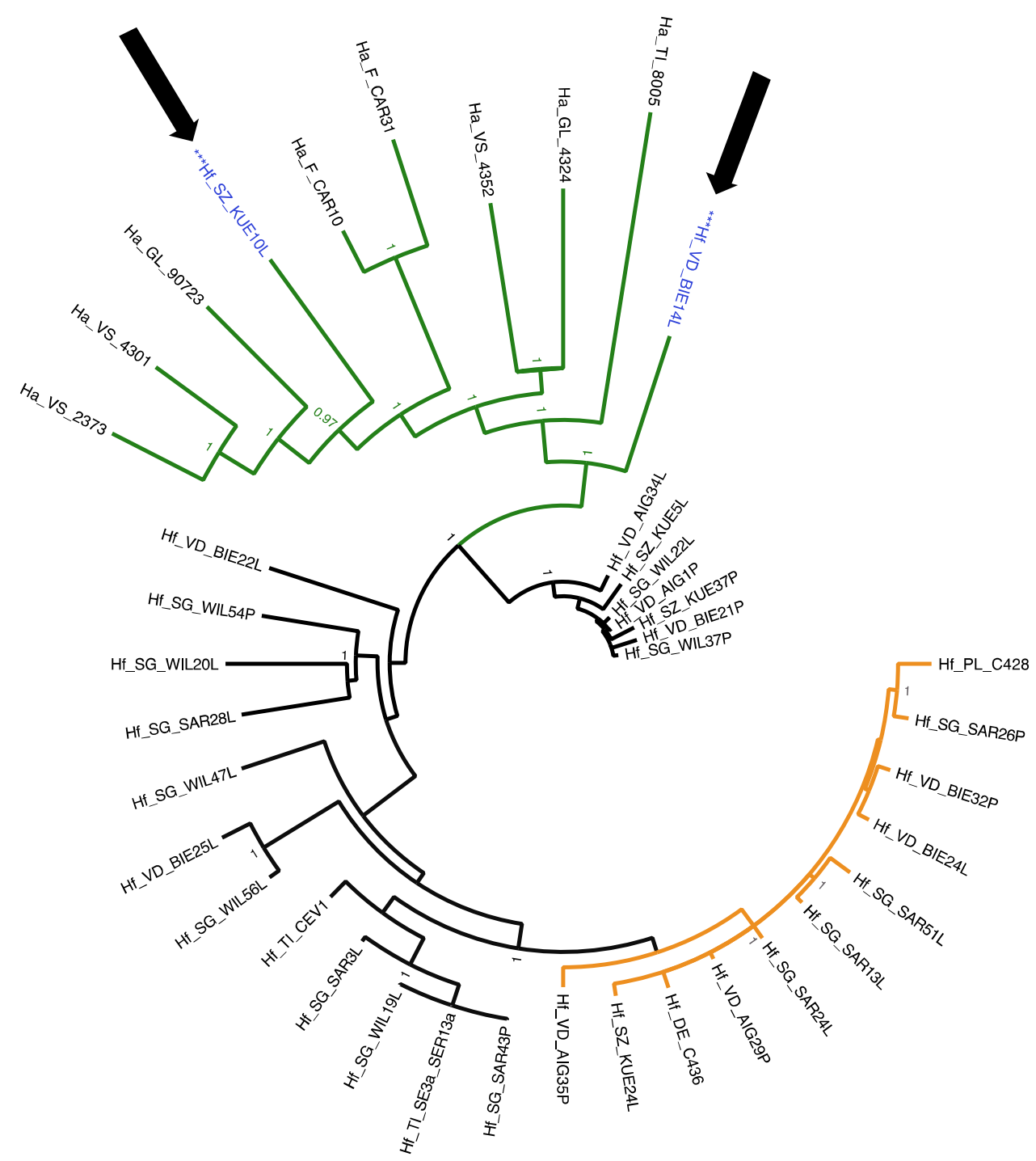

Figure 2. Phylogenetic tree resulting from the MrBayes analysis conducted with $40(8 \mathrm{H}$. albidus, $32 \mathrm{H}$. fraxineus) full sequences (2151 bp) of the RdRP gene of the mitovirus HfMV1. Green color depicts HfMV1 H. albidus group isolates. Black color depicts HfMV1 group 2 isolates and orange color HfMV1 group 1 isolates. Blue color and ${ }^{* * *}$ marks $H$. fraxineus isolates within the $H$. albidus group. $\mathrm{Hf}$ characterizes viral sequences from $H$. fraxineus, $\mathrm{Ha}$ from $H$. albidus isolates. For abbreviation see Table 1. Posterior probabilities are shown at the respective branch if they were $>0.95$. Arrows indicate intermixing of $H$. albidus and $H$. fraxineus HfMV1 sequences.

For the partial RdRP gene of HfMV1 (495 bp), two major groups of sequences ( $\mathrm{PP}=1$, bootstrap value $61 \%$ ) were detected (Figure 3). In accordance with the full RdRP gene sequences and [14] these groups were again called HfMV1 group 1 and HfMV1 group 2. The H. albidus sequences formed three distinct clusters, which were all closer related to HfMV1 group 2 than to HfMV1 group 1 (Figure 3). 


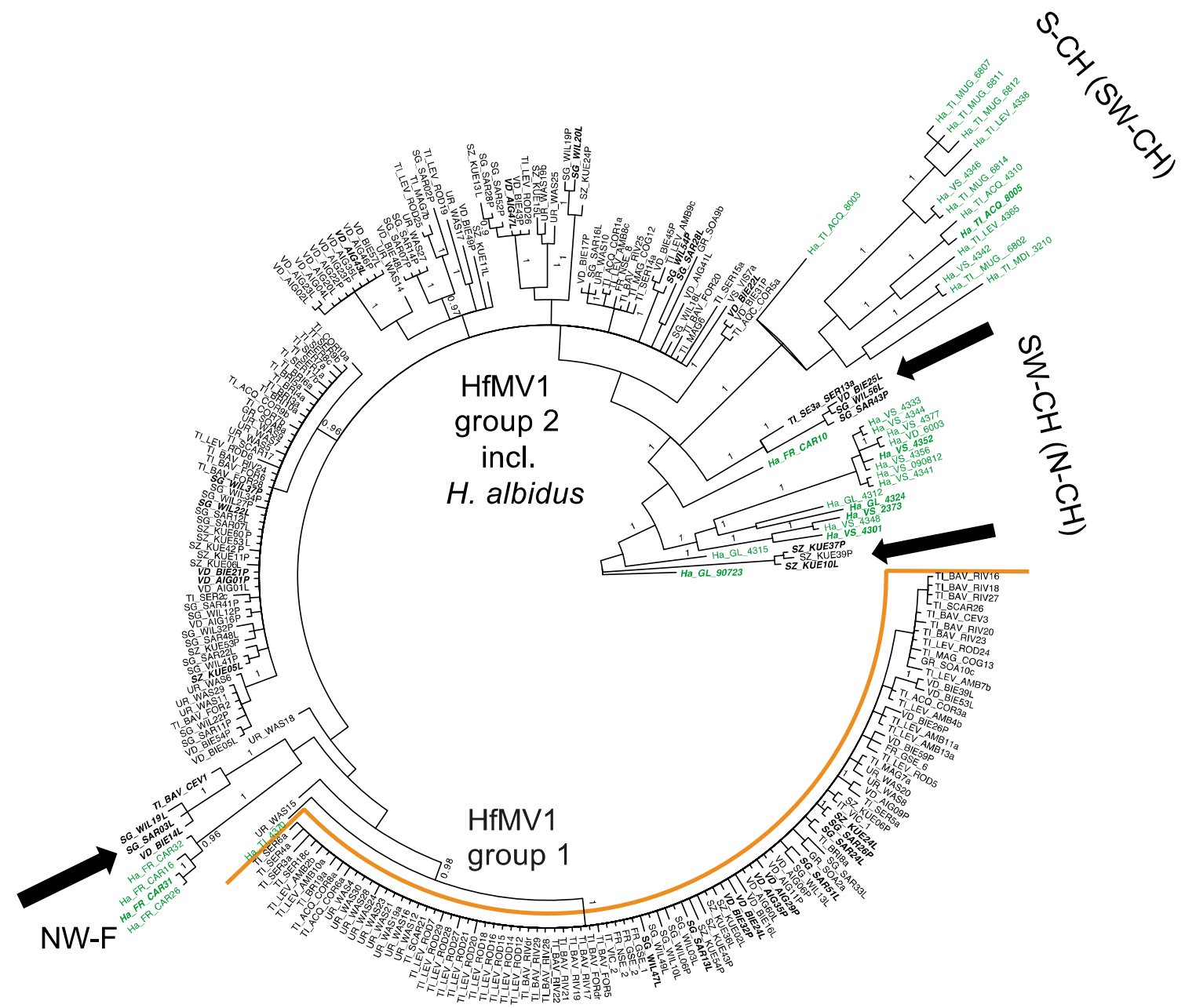

Figure 3. MrBayes analysis conducted with 255 sequences of the partial RdRP gene (495 bp) of the mitovirus HfMV1. Green color depicts H. albidus isolates. Posterior probabilities are shown at the respective branch if they were $>0.95$. Each sample is named with the sampling year, followed by the sampling locality (for abbreviation see Table 1) and sample name. Arrows indicate intermixing of $H$. albidus and $H$. fraxineus HfMV1 sequences. Samples marked in bold and italics are included in the full-length analysis.

No structure could be detected in the overall HfMV1 population (i.e., combining H. albidus and $H$. fraxineus), neither geographically nor chronologically, using area or sampling year as priors. Nonetheless, within HfMV1 sequences of $\mathrm{H}$. albidus, a certain geographic clustering was visible, with French (NW-F) isolates grouping together most distinctly (Figure 3). The 12 HfMV1 sequences from Southern Switzerland, together with two sequences from South-Western Switzerland (SW-CH) formed the second most distinct cluster (Figure 3 and Figure S2). Most sequences from SW-CH also grouped together in a separate cluster. Noteworthy, $12 \mathrm{H}$. fraxineus HfMV1 sequences grouped within the three H. albidus clusters (indicated by black arrows in Figure 3).

\subsection{Population Genetic Parameters}

\subsubsection{Full RdRP Gene}

Given that all full-length RdRP sequences were different, the haplotype diversity (Hd) was one in both $\mathrm{H}$. fraxineus and $\mathrm{H}$. albidus (Table 2). The nucleotide diversity estimated by the average number of differences per site between two sequences $(\pi)$ was 0.03 across all HfMV1 sequences from both host species for the full ORF (2151 bp; $\mathrm{N}=40$; Table 2). Among HfMV1 sequences from 
$H$. albidus, $\pi$ was 0.077 , and 0.021 for $H$. fraxineus sequences. The average number of nucleotide differences $\mathrm{K}$, was 52 across all sequences from both species and ranged between 38 in $H$. fraxineus and 163 in $H$. albidus. The total number of mutations $(\eta)$ was 380 for the entire population $(H$. fraxineus and $H$. albidus combined) and was lower in the Swiss $H$. fraxineus (238) than in the Swiss $H$. albidus population (403; Table 2).

Table 2. Population genetic parameters for the full length RdRP gene sequences (2151 bp) of HfMV1 recovered from Hymenoscyphus albidus and H. fraxineus.

\begin{tabular}{|c|c|c|c|c|c|c|c|c|c|}
\hline Population & $\mathbf{N}$ & Sequence Length & Net Sites & $\mathbf{S}$ & $\eta$ & $\mathbf{h}$ & Hd & $\pi$ & $\mathbf{K}$ \\
\hline H. fraxineus & 32 & 2151 & 1791 & 219 & 239 & 30 & 1.0 & 0.021 & 38 \\
\hline H. albidus & 8 & 2151 & 2122 & 400 & 464 & 8 & 1.0 & 0.077 & 163 \\
\hline H. fraxineus $\mathrm{CH}$ & 30 & 2151 & 1851 & 218 & 238 & 28 & 1.0 & 0.021 & 39 \\
\hline H. albidus $\mathrm{CH}$ & 6 & 2151 & 2129 & 360 & 403 & 6 & 1.0 & 0.076 & 163 \\
\hline H. fraxineus \& $H$. albidus total & 40 & 2151 & 1774 & 328 & 380 & 38 & 1.0 & 0.029 & 52 \\
\hline
\end{tabular}

Abbreviations: N, number of isolates; Sequence length, entire sequence length in bp; Net sites, sequence length in analysis; $S$, number of segregating sites; $\eta$, total number of mutations occurred in that population; $h$, number of haplotypes; $\mathrm{Hd}$, haplotype diversity; $\pi$, nucleotide diversity estimated by the average number of differences per site between two sequences; $\mathrm{K}$, average number of nucleotide differences; $\mathrm{CH}$, only samples from Switzerland (30 out of 32 in $H$. fraxineus and 6 out of 8 in H. albidus).

\subsubsection{Partial RdRP Gene}

For the partial RdRP sequences (495 bp; $\mathrm{N}=255), \pi$ was on average 0.02 (0.08 for $H$. albidus and 0.007 for $H$. fraxineus; Table 3). Hd was on average 0.84 and ranged between 0.90 and 1.00 in $H$. albidus and between 0.7 and 0.99 in $H$. fraxineus. $\mathrm{K}$, the average number of nucleotide differences, was 5 across all sequences from both species and ranged between 3 in $H$. fraxineus and 30 in H. albidus, all from Southern Switzerland (S-CH; Figure 1). The total number of mutations ( $\eta$ ) was 89 for the entire population (H. fraxineus and H. albidus combined) and lower in Swiss $H$. fraxineus (58) than in Swiss H. albidus populations (122; Table 3).

Table 3. Population genetic parameters for the partial RdRP gene sequences (495 bp) of HfMV1 recovered from Hymenoscyphus albidus and $H$. fraxineus.

\begin{tabular}{|c|c|c|c|c|c|c|c|c|c|}
\hline Population & $\mathbf{N}$ & Sequence Length & Net Sites & $\mathbf{S}$ & $\eta$ & $\mathbf{h}$ & Hd & $\pi$ & $\mathbf{K}$ \\
\hline H. fraxineus all & 221 & 495 & 348 & 49 & 57 & 48 & 0.79 & 0.007 & 2.35 \\
\hline H. fraxineus $\mathrm{CH}$ & 214 & 495 & 349 & 50 & 58 & 49 & 0.80 & 0.007 & 2.41 \\
\hline H. fraxineus S-CH * & 90 & 495 & 359 & 33 & 37 & 28 & 0.80 & 0.008 & 2.98 \\
\hline H. fraxineus SW-CH & 41 & 495 & 485 & 89 & 102 & 27 & 0.96 & 0.039 & 19.21 \\
\hline H. fraxineus N-CH & 85 & 495 & 458 & 73 & 81 & 53 & 0.96 & 0.019 & 8.72 \\
\hline H. fraxineus NW-F & 5 & 495 & 488 & 16 & 16 & 3 & 0.70 & 0.014 & 6.60 \\
\hline H. albidus all & 34 & 495 & 426 & 99 & 129 & 28 & 0.99 & 0.080 & 34.02 \\
\hline H. albidus $\mathrm{CH}$ & 29 & 495 & 427 & 94 & 122 & 24 & 0.99 & 0.078 & 33.39 \\
\hline H. albidus S-CH & 12 & 495 & 443 & 79 & 94 & 12 & 1.00 & 0.067 & 29.74 \\
\hline H. albidus SW-CH & 13 & 495 & 467 & 76 & 88 & 10 & 0.96 & 0.052 & 24.03 \\
\hline H. albidus NW-F & 5 & 495 & 494 & 34 & 34 & 4 & 0.90 & 0.026 & 14.40 \\
\hline H. fraxineus \& $H$. albidus total & 255 & 495 & 348 & 71 & 89 & 73 & 0.84 & 0.015 & 5.33 \\
\hline
\end{tabular}

Only populations $\geq 5$ samples were considered. Abbreviations: N, number of isolates; Sequence length, entire sequence length in bp; Net sites, sequence length in analysis; $S$, number of segregating sites; $\eta$, total number of mutations occurred in that population; h, number of haplotypes; Hd, haplotype diversity; $\pi$, nucleotide diversity estimated by the average number of differences per site between two sequences; $K$, average number of nucleotide differences; CH: Switzerland, S-CH: Southern Switzerland, SW-CH: South-Western Switzerland, N-CH: Northern Switzerland, NW-F: North-Western France, * including 2 isolates from Northern Italy.

\section{Discussion}

The main objective of this study was to determine the presence and prevalence of the mitovirus HfMV1 [26] in the leaf endophyte H. albidus and to use the viral RdRP gene for population genetic analyses. Furthermore, we aimed to compare the genetic diversity of HfMV1 in H. albidus, which is presumably native to Europe, to that in the closely related and introduced fungus $H$. fraxineus, 
the causal agent of ash dieback (ADB). Both fungi occupy the same ecological niche, colonizing and infecting ash leaves and forming fruiting bodies on leaf petioles in the litter. While $H$. fraxineus is very abundant, $H$. albidus is now difficult to find and possibly locally extinct $[19,20]$. Our study supports this observation, as from 2013 onwards we could only isolate H. fraxineus even in areas where H. albidus had previously been officially reported. It is generally assumed that this is the result of a displacement of $H$. albidus due to the competition for the sporulation niche with its relative $H$. fraxineus. As $H$. fraxineus acts as a primary leaf pathogen and produces massive airborne spore clouds (see also [21]), it is outcompeting the native $H$. albidus.

Genetic analyses confirmed that the mitovirus detected in H. albidus effectively corresponds to the mitovirus HfMV1 previously described in the invasive pathogen $H$. fraxineus. First, the viral RdRP gene has an identical ORF length in both fungi. Second, the full RdRP gene sequences obtained from $H$. albidus and $H$. fraxineus showed high pairwise identities (94.5\% for the RdRP amino acid sequences, 91.1\% for nucleotide sequences). As this finding indicates that HfMV1 is conspecific in $H$. fraxineus and $H$. albidus, we propose the same virus name, HfMV1, for both fungal species. This is the first description of a fungal virus in the leaf endophyte H. albidus.

The overall prevalence of HfMV1 in H. albidus is lower (49.3\%) than previously described for H. fraxineus isolates from the European mainland and Great Britain $(78.7 \%$ and $67 \%$, respectively; $[15,24])$. This difference could be due to the fact that we analyzed far more $H$. fraxineus compared to H. albidus isolates. Furthermore, there could be a geographic difference, as the H. albidus isolates in this study were obtained mainly from Switzerland (and France), whereas the H. fraxineus isolates were collected throughout Europe (and Great Britain). The lower prevalence of HfMV1 in $H$. albidus could also be due to the PCR assay used for virus screening, which presumably did not detect all HfMV1 variants in this species. Nonetheless, the prevalence of HfMV1 in both Hymenoscyphus species is in accordance with the ranges observed for mitoviruses in other fungal species e.g., in Gremmeniella [43].

All H. albidus isolates analyzed in this study, including those infected by HfMV1, were collected in Switzerland and North-Western France prior to the arrival of ADB (Figure 1; [35]). Therefore, we can assume that HfMV1 must have been present in H. albidus before the introduction of H. fraxineus into Europe. This assumption is supported by the fact that $H$. albidus viruses form a distinct, well supported phylogenetic group when considering the full viral RdRP gene (Figure 2). The H. albidus viruses also exhibit a higher genetic diversity than the $H$. fraxineus viruses. Moreover, clustering by geographic region is evident for HfMV1 strains from H. albidus. Specifically, sequences from Switzerland are clearly separated from those from North-Western France. Within Switzerland, sequences from Southern Switzerland are quite distinct and only partially intermix with HfMV1 sequences from South-Western Switzerland. Such spatial genetic patterns are expected due to local differentiation of HfMV1 in native populations of $H$. albidus. In contrast, we did not detect any geographic structuring for HfMV1 sequences obtained from $H$. fraxineus, which is in accordance with previous studies for the fungus and the virus [12-15]. In summary, these findings suggest that HfMV1 is also native to Europe where it occurs as a genetically distinct group in H. albidus.

The presence of a conspecific mitovirus in $H$. fraxineus and $H$. albidus could be the result of a parallel virus evolution from a common ancestor. Mitoviruses are the most widespread fungal viruses and HfMV1only encodes one single gene. Hence, possibilities for mutations are limited and similar HfMV1 variants may form independently (in parallel) in different fungal hosts, particularly if the host species are closely related. Since mating between the two fungal species, which also possess different mating systems (homothallic in H. albidus vs. heterothallic in H. fraxineus), has never been observed [44], transfer of HfMV1 from H. albidus to H. fraxineus through interspecific hybridization does not seem to be a plausible hypothesis.

The detection of HfMV1 in H. albidus sheds new light on previous research, where only viral sequences obtained from $H$. fraxineus were investigated [14]. Based on the discovery of two HfMV1 groups in Europe, it was hypothesized that two divergent HfMV1 strains were introduced together 
with two $H$. fraxineus strains. The two HfMV1 groups in $H$. fraxineus were also detected in the present study for both the full and partial viral RdRP gene sequences. Although the H. albidus viruses are well separated from these two groups, they seem to be more closely related to HfMV1 group 2 than to group 1 (Figures 2 and 3). Most interestingly, there are several HfMV1 sequences from $H$. fraxineus, which cluster together with $H$. albidus viruses, i.e., these viruses are closer related to those in $H$. albidus than to those in $H$. fraxineus (arrows in Figures 2 and 3). This pattern is consistent with the hypothesis of an interspecies virus transmission from the native $H$. albidus to the invasive $H$. fraxineus. In particular, there are 2 full sequences of $H$. fraxineus isolated from necrotic lesions that cluster with the $H$. albidus group (Figure 2). Our results suggest at least three cross-species transmission events of HfMV1. Further interspecies virus transfers cannot be ruled out particularly within HfMV1 group 2, which could explain the higher genetic diversity observed in this group compared to group 1 [15]. In plant pathogenic fungi, cross-species transmission of mycoviruses has been previously described in different genera, e.g., Cryphonectria hypovirus 1 (family Hypoviridae) in the genus Cryphonectria [30] and the Heterobasidion RNA virus 1 (HetRV1; family Partitiviridae) in the genus Heterobasidion [31]. For HetRV1, Vainio et al. [31] detected high nucleotide level similarity (98\%) between HetRV1 obtained from taxonomically distant $H$. parviporum and $H$. australe, thus suggesting a recent HetRV1 transmission in nature. Furthermore, Deng et al. [33] reported a mitovirus to be conspecific in Sclerotinia homoeocarpa, the causal agent of Dollar spot, and Ophiostoma novo-ulmi, the causal agent of Dutch elm disease, with 92.4\% (nucleotide) and 95.1\% (amino acid) sequence identities between viral strains and [45] detected 95\% identity of BcMV1 and Ophiostoma novo-ulmi mitovirus $3 b$.

In contrast to plant and animal pathogenic viruses, natural vectors are largely unknown in fungal viruses, which typically have no extracellular phase [25]. In vitro experiments (e.g., [30,32]) have shown that mycoviruses are transmitted horizontally between fungal species via hyphal anastomosis. Recent genomic studies revealed that the genomes of $H$. fraxineus and $H$. albidus are highly similar [46] and 75\% of the $H$. albidus reads could be mapped to the $H$. fraxineus reference genome [16]. Hence, hyphal anastomosis and transfer of genetic elements between both fungal species without the final hybridization event cannot be excluded. The low detection rate of such events (i.e., intermixing of HfMV1 sequences from $H$. fraxineus with sequence clusters of HfMV1 from H. albidus) in this study might be simply due to the fact that it is still too early in time to detect them more frequently. Moreover, it has to be kept in mind, that most likely we did not capture the full picture and all transmission events, as only a small number of $H$. albidus isolates from Switzerland and France were investigated in the present study. Future sampling of $H$. fraxineus in areas where divergent HfMV1 strains were found in H. albidus (e.g., Southern Switzerland or North-Western France), as well as the inclusion of additional $H$. albidus samples from other countries could reveal additional evidence of interspecies transmission of HfMV1.

\section{Conclusions}

Our study shows that the mitovirus HfMV1 that was previously identified in the ash dieback pathogen $H$. fraxineus [26] is also occurring in the congeneric species $H$. albidus. Sampling history together with phylogenetic and population genetic analyses suggest that HfMV1 is a native mycovirus in the European $H$. albidus population. The occurrence of a conspecific mitovirus in $H$. albidus and $H$. fraxineus is most likely the result of parallel virus evolution in the two fungal hosts. In addition, our study provides evidence for interspecies virus transmission from $H$. albidus to $H$. fraxineus, which contributed to the viral diversity observed in the invasive $H$. fraxineus population in Europe.

Supplementary Materials: The following are available online at http:/ /www.mdpi.com/1999-4915/10/11/628/ s1, Figure S1: Phylogenetic tree resulting from the RaxML analysis conducted with 40 (8 H. albidus, $32 \mathrm{H}$. fraxineus) full sequences (2151 bp) of the RdRP gene of the mitovirus HfMV1. Green color depicts HfMV1 H. albidus group isolates. Black color depicts HfMV1 group 2 isolates and grey color HfMV1 group isolates. Blue color and *** mark $H$. fraxineus isolates within the $H$. albidus group. Hf characterizes viral sequences from $H$. fraxineus, Ha from $H$. albidus isolates. For abbreviation see Table 1. Bootstrap values are shown if $>70$. Arrows indicate intermixing of H. albidus and H. fraxineus HfMV1 sequences. Figure S2: RAxML analysis conducted with 255 sequences (495 bp) 
of the partial RdRP gene of the mitovirus HfMV1. Each sample is named with the sampling year, followed by the sampling locality (for abbreviation see Table 1) and sample name. Bootstrap values are show if $\geq 60$. Samples marked in bold and italics are included in the full-length analysis. Table S1: Details of the isolates in the study with sample locations, years and GenBank accession numbers. Table S2: List of additional primers used to sequence the full open reading frame of Hymenoscyphus fraxineus mitovirus 1 and $5^{\prime}$ and $3^{\prime}$ flanking regions of HfMV1. Tm = annealing temperature in degrees Celsius.

Author Contributions: Conceptualization, C.N.S., D.R. and S.P.; Methodology, C.N.S., D.R. and S.P.; Formal Analysis, C.N.S.; Investigation, C.N.S.; Resources, D.R. and A.G.; Data Curation, C.N.S.; Writing-Original Draft Preparation, C.N.S.; Writing-Review \& Editing, C.N.S., D.R., A.G. and S.P.; Visualization, C.N.S.; Funding Acquisition, D.R.

Funding: This research was funded by the Lithuanian-Swiss cooperation program to reduce economic and social disparities within the enlarged European Union (project grant agreement no. CH-3-SMM-01/12).

Acknowledgments: We would like to thank O. Holdenrieder, V. Queloz, and Th. Kirisits for providing H. albidus cultures. We are very thankful to E. Jung, S. Pfister, O. Zuberbühler, K. Moosbrugger, J. Schwarz, U. Oggenfuss and C. Cornejo for much appreciated help in the laboratory. We are grateful to the members of the COST Action FP1103 (FRAXBACK) for initiating sample exchange and to Lea Stauber for careful proofreading of the manuscript.

Conflicts of Interest: The authors declare no conflict of interest.

\section{References}

1. Westphal, M.I.; Browne, M.; MacKinnon, K.; Noble, I. The link between international trade and the global distribution of invasive alien species. Biol. Invasions 2008, 10, 391-398. [CrossRef]

2. Santini, A.; Ghelardini, L.; De Pace, C.; Desprez-Loustau, M.L.; Capretti, P.; Chandelier, A.; Cech, T.; Chira, D.; Diamandis, S.; Gaitniekis, T.; et al. Biogeographical patterns and determinants of invasion by forest pathogens in Europe. New Phytol. 2013, 197, 238-250. [CrossRef] [PubMed]

3. Hantula, J.; Mu, M.M.; Uusivuori, J. International plant trade associated risks: Laissez-faire or novel solutions. Environ. Sci. Policy 2014, 37, 158-160. [CrossRef]

4. Grünwald, N.J.; Garbelotto, M.; Goss, E.M.; Heunges, K.; Prospero, S. Emergence of the sudden oak death pathogen Phytophthora ramorum. Trends Microbiol. 2012, 20, 131-138. [CrossRef] [PubMed]

5. Rigling, D.; Prospero, S. Cryphonectria parasitica, the causal agent of chestnut blight: Invasion history, population biology and disease control. Mol. Plant Pathol. 2018, 19, 7-12. [CrossRef] [PubMed]

6. Hoegger, P.J.; Rigling, D.; Holdenrieder, O.; Heiniger, U. Cryphonectria radicalis: Rediscovery of a lost fungus. Mycologia 2002, 94, 105-115. [CrossRef] [PubMed]

7. Gonthier, P.; Nicolotti, G.; Linzer, R.; Guglielmo, F.; Garbelotto, M. Invasion of European pine stands by a North American forest pathogen and its hybridization with a native interfertile taxon. Mol. Ecol. 2007, 16, 1389-1400. [CrossRef] [PubMed]

8. Érsek, T.; Nagy, Z.A. Species hybrids in the genus Phytophthora with emphasis on the alder pathogen Phytophthora alni: A review. Eur. J. Plant Pathol. 2008, 122, 31-39. [CrossRef]

9. Gross, A.; Han, J.G. Hymenoscyphus fraxineus and two new Hymenoscyphus species identified in Korea. Mycol. Prog. 2015, 14, 1. [CrossRef]

10. Gross, A.; Zaffarano, P.L.; Duo, A.; Grünig, C.R. Reproductive mode and life-cycle of the ash dieback pathogen Hymenoscyphus pseudoalbidus. Fungal Genet. Biol. 2012, 49, 977-986. [CrossRef] [PubMed]

11. Baral, H.O.; Bemmann, M. Hymenoscyphus fraxineus vs. Hymenoscyphus albidus-A comparative light microscopic study on the causal agent of European ash dieback and related foliicolous, stroma-forming species. Mycology 2014, 5, 228-290. [PubMed]

12. Bengtsson, S.B.K.; Vasaitis, R.; Kirisits, T.; Solheim, H.; Stenlid, J. Population structure of Hymenoscyphus pseudoalbidus and its genetic relationship to Hymenoscyphus albidus. Fungal Ecol. 2012, 5, 147-153. [CrossRef]

13. Burokiene, D.; Prospero, S.; Jung, E.; Marciulyniene, D.; Moosbrugger, K.; Norkute, G.; Rigling, D.; Lygis, V.; Schoebel, C.N. Genetic population structure of the invasive ash dieback pathogen Hymenoscyphus fraxineus in its expanding range. Biol. Invasions 2015, 17, 2743-2756. [CrossRef]

14. Gross, A.; Hosoya, T.; Queloz, V. Population structure of the invasive forest pathogen Hymenoscyphus pseudoalbidus. Mol. Ecol. 2014, 23, 2943-2960. [CrossRef] [PubMed]

15. Schoebel, C.N.; Botella, L.; Lygis, V.; Rigling, D. Population genetic analysis of a parasitic mycovirus to infer the invasion history of its fungal host. Mol. Ecol. 2017, 26, 2482-2497. [CrossRef] [PubMed] 
16. Sonstebo, J.H.; Smith, A.V.; Adamson, K.; Drenkhan, R.; Solheim, H.; Hietala, A. Genome-wide population diversity in Hymenoscyphus fraxineus points to an eastern Russian origin of European Ash dieback. bioRxiv 2017, 154492. [CrossRef]

17. Kirisits, T.; Dämpfle, L.; Kräutler, K. Hymenoscyphus albidus is not associated with an anamorphic stage and displays slower growth than Hymenoscyphus pseudoalbidus on agar media. For. Pathol. 2013, 43, 386-389.

18. Brasier, C.; King, K.; Kirisits, T.; Orton, E.; Webber, J. High frequency of vegetative incompatibility combined with haploid selfing in the native European ash foliage coloniser Hymenoscyphus albidus. Fungal Ecol. 2017, 28, 11-24. [CrossRef]

19. McKinney, L.V.; Thomsen, I.M.; Kjær, D.; Bengtsson, S.B.K.; Nielsen, L.R. Rapid invasion by an aggressive pathogenic fungus (Hymenoscyphus pseudoalbidus) replaces a native decomposer (Hymenoscyphus albidus): A case of local cryptic extinction? Fungal Ecol. 2012, 5, 663-669. [CrossRef]

20. King, K.M.; Webber, J.F. Development of a multiplex PCR assay to discriminate native Hymenoscyphus albidus and introduced Hymenoscyphus fraxineus in Britain and assess their distribution. Fungal Ecol. 2016, 23, 79-85. [CrossRef]

21. Hietala, A.M.; Timmermann, V.; Barja, I.; Solheim, H. The invasive ash dieback pathogen Hymenoscyphus pseudoalbidus exerts maximal infection pressure prior to the onset of host leaf senescence. Fungal Ecol. 2013, 6, 302-308. [CrossRef]

22. Kowalski, T.; Holdenrieder, O. Pathogenicity of Chalara fraxinea. For. Pathol. 2009, 39, 1-7. [CrossRef]

23. Pautasso, M.; Aas, G.; Queloz, V.; Holdenrieder, O. European ash (Fraxinus excelsior) dieback-A conservation biology challenge. Biol. Conserv. 2013, 158, 37-49. [CrossRef]

24. Orton, E.S.; Brasier, C.M.; Bilham, L.J.; Bansal, A.; Webber, J.F.; Brown, J.K.M. Population structure of the ash dieback pathogen, Hymenoscyphus fraxineus, in relation to its mode of arrival in the UK. Plant Pathol. 2018, 67, 255-264. [CrossRef] [PubMed]

25. Ghabrial, S.A.; Suzuki, N. Viruses of plant pathogenic fungi. Annu. Rev. Phytopathol. 2009, 47, $353-384$. [CrossRef] [PubMed]

26. Schoebel, C.N.; Zoller, S.; Rigling, D. Detection and genetic characterization of a novel mycovirus in Hymenoscyphus fraxineus, the causal agent of ash dieback. Infect. Genet. Evol. 2014, 28, 78-86. [CrossRef] [PubMed]

27. Li, W.H. Molecular Evolution; Sinauer Associates, Inc.: Sunderland, MA, USA, 1997.

28. Polashock, J.J.; Hillman, B.I. A small mitochondrial double-stranded (ds) RNA element associated with a hypovirulent strain of the chestnut blight fungus and ancestrally related to yeast cytoplasmic $\mathrm{T}$ and $\mathrm{W}$ dsRNAs. Proc. Natl. Acad. Sci. USA 1994, 91, 8680-8684. [CrossRef] [PubMed]

29. Polashock, J.; Bedker, P.; Hillman, B. Movement of a small mitochondrial double-stranded RNA element of Cryphonectria parasitica: Ascospore inheritance and implications for mitochondrial recombination. Mol. Gen. Genet. 1997, 256, 566-571. [CrossRef] [PubMed]

30. Liu, Y.C.; Linder-Basso, D.; Hillman, B.I.; Kaneko, S.; Milgroom, M.G. Evidence for interspecies transmission of viruses in natural populations of filamentous fungi in the genus Cryphonectria. Mol. Ecol. 2003, 12, 1619-1628. [CrossRef] [PubMed]

31. Vainio, E.J.; Hakanpää, J.; Dai, Y.C.; Hansen, E.; Korhonen, K.; Hantula, J. Species of Heterobasidion host a diverse pool of partitiviruses with global distribution and interspecies transmission. Fungal Biol. 2011, 115, 1234-1243. [CrossRef] [PubMed]

32. Melzer, M.; Ikeda, S.S.; Boland, G.J. Interspecific transmission of double-stranded RNA and hypovirulence from Sclerotinia sclerotiorum to S. minor. Phytopathology 2002, 92, 780-784. [CrossRef] [PubMed]

33. Deng, F.; Xu, R.; Boland, G. Hypovirulence-associated double-stranded RNA from Sclerotinia homoeocarpa is conspecific with Ophiostoma novo-ulmi mitovirus 3a-Ld. Phytopathology 2003, 93, 1407-1414. [CrossRef] [PubMed]

34. White, T.; Bruns, T.; Lee, S.; Taylor, J. Amplification and direct sequencing of fungal ribosomal RNA genes for phylogenetics. In PCR Protocols: A Guide to Methods and Applications; Innis, M., Gelfand, D., Sninsky, J., White, T., Eds.; Academic Press: New York, NY, USA, 1990; pp. 315-322. [CrossRef]

35. Queloz, V.; Grünig, C.R.; Berndt, R.; Berndt, R.; Kowalski, T.; Sieber, T.N.; Holdenrieder, O. Cryptic speciation in Hymenoscyphus albidus. For. Pathol. 2011, 41, 133-142. [CrossRef]

36. Ranwez, V.; Harispe, S.; Delsuc, F.; Douzery, E.J.P. MACSE: Multiple Alignment of Coding Sequences accounting for frameshifts and stop codons. PLoS ONE 2011, 6, e22594. [CrossRef] [PubMed] 
37. Stamatakis, A. RAxML-VI-HPC: Maximum likelihood-based phylogenetic analyses with thousands of taxa and mixed models. Bioinformatics 2006, 22, 2688-2690. [CrossRef] [PubMed]

38. Ronquist, F.; Teslenko, M.; Van der Mark, P.; Ayres, D.L.; Darling, A.; Höhna, S.; Larget, B.; Liu, L.; Suchard, M.A.; Huelsenbeck, J.P. MrBayes 3.2: Efficient Bayesian phylogenetic inference and model choice across a large model space. Syst. Biol. 2012, 61, 539-542. [CrossRef] [PubMed]

39. Stöver, B.C.; Müller, K.F. TreeGraph 2: Combining and visualizing evidence from different phylogenetic analyses. BMC Bioinform. 2010, 11, 7. [CrossRef] [PubMed]

40. Librado, P.; Rozas, J. DnaSP v5: A software for comprehensive analysis of DNA polymorphism data. Bioinformatics 2009, 25, 1451-1452. [CrossRef] [PubMed]

41. Rigling, D.; Hilfiker, S.; Schöbel, C.; Meier, F.; Engesser, R.; Scheidegger, C.; Stofer, S.; Senn-Irlet, B.; Queloz, V. Das Eschentriebsterben: Biologie, Krankheitssymptome und Handlungsempfehlungen; Merkbl. Publisher: Birmensdorf, Switzerland, 2016.

42. Buck, K.W.; Esteban, R.; Hillman, B.I. Narnaviridae. In Virus Taxonomy: Eighth Report of the International Committee on Taxonomy of Viruses, 1st ed.; Fauquet, C.M., Mayo, M.A., Maniloff, J., Desselberger, U., Ball, L.A., Eds.; Elsevier Academic Press: San Diego, CA, USA, 2005; pp. 751-756.

43. Botella, L.; Tuomivirta, T.T.; Vervuurt, S.; Diez, J.J.; Hantula, J. Occurrence of two different species of mitoviruses in the European race of Gremmeniella abietina var. abietina, both hosted by the genetically unique Spanish population. Fungal Biol. 2012, 116, 872-882. [PubMed]

44. Wey, T.; Schlegel, M.; Stroheker, S.; Gross, A. MAT-gene structure and mating behavior of Hymenoscyphus fraxineus and Hymenoscyphus albidus. Fungal Genet. Biol. 2016, 87, 54-63. [CrossRef] [PubMed]

45. Wu, M.; Zhang, L.; Li, G.; Jiang, D.; Ghabrial, S.A. Genome characterization of a debilitation-associated mitovirus infecting the phytopathogenic fungus Botrytis cinereal. Virology 2010, 406, 117-126. [CrossRef] [PubMed]

46. Stenlid, J.; Elfstand, M.; Cleary, M.; Ihrmark, K.; Karlsson, M.; Davydenko, K.; Brandström Durling, M. Genomes of Hymenoscyphus fraxineus and Hymenoscyphus albidus Encode Surprisingly Large Cell Wall Degrading Potential, Balancing Saprotrophic and Necrotrophic Signatures. Baltic For. 2017, 23, 89-106. 